\title{
Fixed point theorems for Meir-Keeler type mappings in $M$-metric spaces with applications
}

Mehdi Asadi*

\section{"Correspondence:}

masadi.azu@gmail.com

Department of Mathematics,

Zanjan Branch, Islamic Azad

University, Zanjan, Iran

\section{Springer}

\begin{abstract}
In this paper, we establish some fixed point theorems for a Meir-Keeler type contraction in M-metric spaces via Gupta-Saxena type contraction. Also, we extend and improve very recent results in fixed point theory.
\end{abstract}

MSC: Primary $47 \mathrm{H} 10$; secondary $54 \mathrm{H} 25$

Keywords: fixed point; partial metric space; $M$-metric space

\section{Introduction and preliminaries}

Ekeland formulated a variational principle that is the foundation of modern variational calculus, having applications in many branches of mathematics, including optimization and fixed point theory [1] and applications in nonlinear analysis, since it entails the existence of approximate solutions of minimization problems for a lower semi-continuous function that is bounded from below on complete metric spaces. Also, Ekeland's variational principle is a fruitful tool in simplifying and unifying the proofs of already known theorems and has many generalizations; see Borwein and Zhu [2].

Matthews in 1994 [3] introduced a partial metric space and proved the contraction principle of Banach in this new framework. Afterward, by several mathematicians many fixed point theorems were founded in partial metric spaces. Recently Haghi et al. [4] published a paper which stated that we should 'be careful on partial metric fixed point results' along with very some results therein. They showed that fixed point generalizations to partial metric spaces can be obtained from the corresponding results in metric spaces.

In 2014, Asadi et al. [5] introduced the $M$-metric space, which extends the $p$-metric space and certain fixed point theorems obtained therein.

In this paper, we establish some of the fixed point theorem for a Meir-Keeler type contraction in $M$-metric spaces via a Gupta-Saxena type contraction. Also, we extend and improve very recent results in fixed point theory.

Definition 1.1 ([3], [6], Definition 1.1) A partial metric on a nonempty set $X$ is a function $p: X \times X \rightarrow \mathbb{R}^{+}$such that for all $x, y, z \in X$ :

(p1) $p(x, x)=p(y, y)=p(x, y) \Longleftrightarrow x=y$,

(p2) $p(x, x) \leq p(x, y)$,

(c) 2015 Asadi. This article is distributed under the terms of the Creative Commons Attribution 4.0 International License (http://creativecommons.org/licenses/by/4.0/), which permits unrestricted use, distribution, and reproduction in any medium, provided you give appropriate credit to the original author(s) and the source, provide a link to the Creative Commons license, and indicate if changes were made. 
(p3) $p(x, y)=p(y, x)$

(p4) $p(x, y) \leq p(x, z)+p(z, y)-p(z, z)$.

A partial metric space is a pair $(X, p)$ such that $X$ is a nonempty set and $p$ is a partial metric on $X$.

Notation The following notations are useful in the sequel:

(i) $m_{x y}:=\min \{m(x, x), m(y, y)\}=m(x, x) \vee m(y, y)$,

(ii) $M_{x y}:=\max \{m(x, x), m(y, y)\}=m(x, x) \wedge m(y, y)$.

Now we want to extend Definition 1.1 as follows.

Definition 1.2 Let $X$ be a non-empty set. A function $m: X \times X \rightarrow \mathbb{R}^{+}$is called a $m$-metric if the following conditions are satisfied:

(m1) $m(x, x)=m(y, y)=m(x, y) \Longleftrightarrow x=y$,

(m2) $m_{x y} \leq m(x, y)$,

(m3) $m(x, y)=m(y, x)$,

(m4) $\left(m(x, y)-m_{x y}\right) \leq\left(m(x, z)-m_{x z}\right)+\left(m(z, y)-m_{z y}\right)$.

Then the pair $(X, m)$ is called an $M$-metric space.

According to the above, our definition of the condition (p1) in the definition [3] changes to (m1) and (p2) for $p(x, x)$ is expressed by just $p(y, y)=0$; we may have $p(y, y) \neq 0$, so we improved that condition by replacing it by $\min \{p(x, x), p(y, y)\} \leq p(x, y)$, and also we improved the condition (p4) to the form (m4). In the sequel we present an example that holds for the $m$-metric, but not for the $p$-metric.

Remark 1.1 For every $x, y \in X$ :

(i) $0 \leq M_{x y}+m_{x y}=m(x, x)+m(y, y)$,

(ii) $0 \leq M_{x y}-m_{x y}=|m(x, x)-m(y, y)|$,

(iii) $M_{x y}-m_{x y} \leq\left(M_{x z}-m_{x z}\right)+\left(M_{z y}-m_{z y}\right)$.

The next examples state that $m^{s}$ and $m^{w}$ are ordinary metrics.

Example 1.1 Let $m$ be a $m$-metric. Put:

(i) $m^{w}(x, y)=m(x, y)-2 m_{x y}+M_{x y}$,

(ii) $m^{s}(x, y)=m(x, y)-m_{x y}$ when $x \neq y$ and $m^{s}(x, y)=0$ if $x=y$.

Then $m^{w}$ and $m^{s}$ are ordinary metrics.

Proof If $m^{w}(x, y)=0$, then

$$
m(x, y)=2 m_{x y}-M_{x y} .
$$

But from (1) and $m_{x y} \leq m(x, y)$ we get $m_{x y}=M_{x y}=m(x, x)=m(y, y)$, so by (1) we obtain $m(x, y)=m(x, x)=m(y, y)$, and therefore $x=y$. For the triangle inequality it is enough that we consider Remark 1.1 and (m4).

In the following example, we present an example of a $m$-metric which is not a $p$-metric. 
Remark 1.2 For every $x, y \in X$ :

(i) $m(x, y)-M_{x y} \leq m^{w}(x, y) \leq m(x, y)+M_{x y}$,

(ii) $\left(m(x, y)-M_{x y}\right) \leq m^{s}(x, y) \leq m(x, y)$.

Example 1.2 Let $X=\{1,2,3\}$. Define

$$
\begin{aligned}
& m(1,2)=m(2,1)=m(1,1)=8, \\
& m(1,3)=m(3,1)=m(3,2)=m(2,3)=7, \quad m(2,2)=9, \quad \text { and } \quad m(3,3)=5,
\end{aligned}
$$

so $m$ is an $m$-metric but $m$ is not a $p$-metric. Since $m(2,2) \not \leq m(1,2), m$ is not a $p$-metric. If $D(x, y)=m(x, y)-m_{x, y}$ then $m(1,2)=m_{1,2}=8$ but it means $D(1,2)=0$, while $1 \neq 2$ means $D$ is not a metric.

Example 1.3 ([5]) Let $(X, d)$ be a metric space, $\phi:[0, \infty) \rightarrow[\phi(0), \infty)$ be a one to one and nondecreasing or strictly increasing mapping with $\phi(0)$, defined such that

$$
\phi(x+y) \leq \phi(x)+\phi(y)-\phi(0) \quad \forall x, y \geq 0 .
$$

Then $m(x, y)=\phi(d(x, y))$ is an $m$-metric.

Example 1.4 Let $(X, d)$ be a metric space. Then $m(x, y)=a d(x, y)+b$ where $a, b>0$ is an $m$-metric, because we can put $\phi(t)=a t+b$.

Remark 1.3 According to the Example 1.4, by the Banach contraction

$$
\exists k \in[0,1), \quad m(T x, T y) \leq k m(x, y), \quad \text { for all } x, y \in X,
$$

we have

$$
m(T x, T y)=a d(T x, T y)+b \leq k a d(x, y)+k b \Rightarrow d(T x, T y) \leq k d(x, y)+\frac{b(k-1)}{a},
$$

which does not imply that we have the ordinary Banach contraction

$$
\exists k \in[0,1), \quad d(T x, T y) \leq k d(x, y) \quad \text { for all } x, y \in X,
$$

for all self-maps $T$ on $X$. So this states that if the $m$-metric $m$ and the ordinary metric $d$ even have the same topology, but the Banach contraction of an $m$-metric, this does not imply the Banach contraction of the ordinary metric $d$.

Lemma 1.1 ([5]) Every p-metric is an m-metric.

\section{Topology for $M$-metric space}

It is clear that each $m$-metric $p$ on $\mathrm{X}$ generates a $T_{0}$ topology $\tau_{m}$ on $X$. The set

$$
\left\{B_{m}(x, \varepsilon): x \in X, \varepsilon>0\right\}
$$


where

$$
B_{m}(x, \varepsilon)=\left\{y \in X: m(x, y)<m_{x, y}+\varepsilon\right\},
$$

for all $x \in X$ and $\varepsilon>0$, forms the base of $\tau_{m}$.

Definition 2.1 Let $(X, m)$ be an $M$-metric space. Then:

(1) A sequence $\left\{x_{n}\right\}$ in an $M$-metric space $(X, m)$ converges to a point $x \in X$ if and only if

$$
\lim _{n \rightarrow \infty}\left(m\left(x_{n}, x\right)-m_{x_{n}, x}\right)=0 .
$$

(2) A sequence $\left\{x_{n}\right\}$ in an $M$-metric space $(X, m)$ is called an $m$-Cauchy sequence if

$$
\lim _{n, m \rightarrow \infty}\left(m\left(x_{n}, x_{m}\right)-m_{x_{n}, x_{m}}\right) \text { and } \lim _{n, m \rightarrow \infty}\left(M_{x_{n}, x_{m}}-m_{x_{n}, x_{m}}\right)
$$

in this space exist (and are finite).

(3) An $M$-metric space $(X, m)$ is said to be complete if every $m$-Cauchy sequence $\left\{x_{n}\right\}$ in $X$ converges, with respect to $\tau_{m}$, to a point $x \in X$ such that

$$
\left(\lim _{n \rightarrow \infty}\left(m\left(x_{n}, x\right)-m_{x_{n}, x}\right)=0 \text { and } \lim _{n \rightarrow \infty}\left(M_{x_{n}, x}-m_{x_{n}, x}\right)=0\right) .
$$

Lemma 2.1 Let $(X, m)$ be an M-metric space. Then:

(1) $\left\{x_{n}\right\}$ is a $m$-Cauchy sequence in $(X, m)$ if and only if it is a Cauchy sequence in the metric space $\left(X, m^{w}\right)$.

(2) An M-metric space $(X, m)$ is complete if and only if the metric space $\left(X, m^{w}\right)$ is complete. Furthermore,

$$
\lim _{n \rightarrow \infty} m^{w}\left(x_{n}, x\right)=0 \Longleftrightarrow\left(\lim _{n \rightarrow \infty}\left(m\left(x_{n}, x\right)-m_{x_{n}, x}\right)=0 \text { and } \lim _{n \rightarrow \infty}\left(M_{x_{n}, x}-m_{x_{n}, x}\right)=0\right) .
$$

Likewise the above definition holds also for $m^{s}$.

Lemma 2.2 Assume that $x_{n} \rightarrow x$ and $y_{n} \rightarrow y$ as $n \rightarrow \infty$ in an $M$-metric space $(X, m)$. Then

$$
\lim _{n \rightarrow \infty}\left(m\left(x_{n}, y_{n}\right)-m_{x_{n}, y_{n}}\right)=m(x, y)-m_{x y} .
$$

Proof We have

$$
\left|\left(m\left(x_{n}, y_{n}\right)-m_{x_{n}, y_{n}}\right)-\left(m(x, y)-m_{x, y}\right)\right| \leq\left(m\left(x_{n}, x\right)-m_{x_{n}, x}\right)+\left(m\left(y, y_{n}\right)-m_{y, y_{n}}\right) .
$$

From Lemma 2.2 we can deduce the following lemma.

Lemma 2.3 Assume that $x_{n} \rightarrow x$ as $n \rightarrow \infty$ in an M-metric space $(X, m)$. Then

$$
\lim _{n \rightarrow \infty}\left(m\left(x_{n}, y\right)-m_{x_{n}, y}\right)=m(x, y)-m_{x, y}
$$

for all $y \in X$. 
Lemma 2.4 Assume that $x_{n} \rightarrow x$ and $x_{n} \rightarrow y$ as $n \rightarrow \infty$ in an M-metric space $(X, m)$. Then $m(x, y)=m_{x y}$. Further if $m(x, x)=m(y, y)$, then $x=y$.

Proof By Lemma 2.2 we have

$$
0=\lim _{n \rightarrow \infty}\left(m\left(x_{n}, x_{n}\right)-m_{x_{n}, x_{n}}\right)=m(x, y)-m_{x y}
$$

Lemma 2.5 Let $\left\{x_{n}\right\}$ be a sequence in an $M$-metric space $(X, m)$, such that

$$
\exists r \in[0,1) \quad \text { such that } m\left(x_{n+1}, x_{n}\right) \leq r m\left(x_{n}, x_{n-1}\right) \quad \forall n \in \mathbb{N} .
$$

Then

(A) $\lim _{n \rightarrow \infty} m\left(x_{n}, x_{n-1}\right)=0$,

(B) $\lim _{n \rightarrow \infty} m\left(x_{n}, x_{n}\right)=0$,

(C) $\lim _{m, n \rightarrow \infty} m_{x_{m} x_{n}}=0$,

(D) $\left\{x_{n}\right\}$ is an $m$-Cauchy sequence.

Proof From (4) we have,

$$
m\left(x_{n}, x_{n-1}\right) \leq r m\left(x_{n-1}, x_{n-2}\right) \leq r^{2} m\left(x_{n-2}, x_{n-3}\right) \leq \cdots \leq r^{n} m\left(x_{0}, x_{1}\right),
$$

thus

$$
\lim _{n \rightarrow \infty} m\left(x_{n}, x_{n-1}\right)=0
$$

which implies (A).

To prove (B), from (m2) and (A) we have

$$
\lim _{n \rightarrow \infty} \min \left\{m\left(x_{n}, x_{n}\right), m\left(x_{n-1}, x_{n-1}\right)\right\}=\lim _{n \rightarrow \infty} m_{x_{n} x_{n-1}} \leq \lim _{n \rightarrow \infty} m\left(x_{n}, x_{n-1}\right)=0 .
$$

That is, (B) holds.

Clearly, (C) holds, since $\lim _{n \rightarrow \infty} m\left(x_{n}, x_{n}\right)=0$.

Theorem 2.1 The topology $\tau_{m}$ is not Hausdorff.

Theorem 2.2 Let $(X, m)$ be a complete $M$-metric space and $T: X \rightarrow X$ be mapping satisfying the following condition:

$$
\exists k \in[0,1) \quad \text { such that } \quad m(T x, T y) \leq k m(x, y) \quad \forall x, y \in X
$$

Then T has a unique fixed point.

Theorem 2.3 Let $(X, m)$ be a complete $M$-metric space and $T: X \rightarrow X$ be mapping satisfying the following condition:

$$
\exists k \in\left[0, \frac{1}{2}\right) \quad \text { such that } \quad m(T x, T y) \leq k(m(x, T x)+m(y, T y)) \quad \forall x, y \in X
$$

Then T has a unique fixed point. 


\section{Main result and fixed point theorems}

The following definition is new version of the definition in [7] for an $M$-metric space.

Definition 3.1 A Meir-Keeler mapping is a mapping $T: M \rightarrow M$ on an $M$-metric space $(X, M)$ such that

$$
\forall \varepsilon>0 \exists \delta>0 \text { such that } \forall x, y \in X \text { and } \varepsilon \leq m(x, y)<\varepsilon+\delta \Rightarrow m(T x, T y)<\varepsilon .
$$

Theorem 3.1 Let $(X, m)$ be a complete $M$-metric space and let $T$ be a mapping from $X$ into itself satisfying the following condition:

$$
\forall \varepsilon>0 \exists \delta>0 \forall x, y \in X \varepsilon \leq m(x, y)<\varepsilon+\delta \Rightarrow m(T x, T y)<\varepsilon .
$$

Then $T$ has a unique fixed point $u \in X$. Moreover, for all $x \in X$, the sequence $\left\{T_{n}(x)\right\}$ converges to $u$.

Proof We first observe that (7) trivially implies that $T$ is a strict contraction, i.e.,

$$
x \neq y \Rightarrow m(T x, T y)<m(x, y) .
$$

Let $x_{0} \in X$ and $x_{n}:=T x_{n-1}$, so we have

$$
m\left(x_{n}, x_{n-1}\right)=m\left(T x_{n-1}, T x_{n-2}\right) \leq m\left(x_{n-1}, x_{n-2}\right) .
$$

So the sequence $\left\{m\left(x_{n}, x_{n-1}\right)\right\}$ is bounded below and decreasing; thus $m\left(x_{n}, x_{n-1}\right) \rightarrow m$ for some $m \in \mathbb{R}^{+}$. Let $m>0$, therefore $m\left(x_{n}, x_{n-1}\right) \geq m$. On the other hand for $m>0$ there exists $\delta(m)>0$ such that

$$
m \leq m\left(x_{n-1}, x_{n-2}\right)<m+\delta(m) \Rightarrow m\left(T x_{n-1}, T x_{n-2}\right)=m\left(x_{n}, x_{n-1}\right)<m,
$$

which implies that it is contradiction; so $m=0$, i.e.,

$$
\begin{aligned}
& \lim _{n \rightarrow \infty} m\left(x_{n}, x_{n+1}\right)=0, \\
& \lim _{n \rightarrow \infty} \min \left\{m\left(x_{n}, x_{n}\right), m\left(x_{n-1}, x_{n-1}\right)\right\}=\lim _{n \rightarrow \infty} m_{x_{n} x_{n-1}} \leq \lim _{n \rightarrow \infty} m\left(x_{n}, x_{n-1}\right)=0,
\end{aligned}
$$

and

$$
\lim _{m, n \rightarrow \infty} m_{x_{m} x_{n}}=0 \text { and } \lim _{m, n \rightarrow \infty} M_{x_{m} x_{n}}=0
$$

since, $\lim _{n \rightarrow \infty} m\left(x_{n}, x_{n}\right)=0$. Now we want to show that $\lim _{m, n \rightarrow \infty} m\left(x_{m}, x_{n}\right)=0$. Let it be untrue. So for some $\varepsilon>0$ we have $\limsup _{m, n \rightarrow \infty} m\left(x_{m}, x_{n}\right)>2 \varepsilon$. Also, by hypothesis, there exists a $\delta>0$, such that

$$
\varepsilon \leq m(x, y)<\varepsilon+\delta \Rightarrow m(T x, T y)<\varepsilon
$$


which remains true with $\delta$ replaced by $\delta^{\prime}=\min \{\delta, \varepsilon\}$. Now by (10)

$$
\exists N>0 \forall n \quad\left(n>N \Rightarrow m\left(x_{n}, x_{n+1}\right)<\frac{\delta^{\prime}}{3}\right)
$$

and for $m, n>N, m\left(x_{m}, x_{n}\right)>2 \varepsilon$. This implies, since

$$
m\left(x_{n}, x_{n+1}\right)<\varepsilon \quad \text { and } \quad \varepsilon+\delta^{\prime}<2 \varepsilon<m\left(x_{m}, x_{n}\right)
$$

that there exists $i$ with $m<i<n$ with

$$
\varepsilon+\frac{2 \delta^{\prime}}{3}<m\left(x_{m}, x_{i}\right)-m_{x_{m}, x_{i}}<\varepsilon+\delta^{\prime}
$$

However, for all $m$ and $i$,

$$
\begin{aligned}
m\left(x_{m}, x_{i}\right)-m_{x_{m}, x_{i}} \leq & m\left(x_{m}, x_{m+1}\right)-m_{x_{m+1}, x_{i+1}}+m\left(x_{m+1}, x_{i+1}\right)-m_{x_{m+1}, x_{i+1}} \\
& +m\left(x_{i+1}, x_{i}\right)-m_{x_{i+1}, x_{i}} \\
\leq & m\left(x_{m}, x_{m+1}\right)+m\left(x_{m+1}, x_{i+1}\right)+m\left(x_{i+1}, x_{i}\right) \\
\leq & \frac{\delta^{\prime}}{3}+\varepsilon+\frac{\delta^{\prime}}{3}
\end{aligned}
$$

which contradicts (12). So by (11) and $\lim _{m, n \rightarrow \infty} m\left(x_{m}, x_{n}\right)=0$ we see that the sequence $\left\{x_{n}\right\}$ is a Cauchy sequence and by completeness of $X, x_{n} \rightarrow x^{*}$ in $m$ for some $x^{*} \in X$, i.e.,

$$
\lim _{n \rightarrow \infty}\left(m\left(x_{n}, x^{*}\right)-m_{x_{n}, x^{*}}\right)=0
$$

But $m_{x_{n}, x^{*}} \rightarrow 0$ because $m\left(x_{n}, x_{n}\right) \rightarrow 0$ so $m\left(x_{n}, x^{*}\right) \rightarrow 0$. Thus, by hypothesis, $m\left(T x_{n}\right.$, $\left.T x^{*}\right) \leq m\left(x_{n}, x^{*}\right) \rightarrow 0$. Hence by $(\mathrm{m} 2) m_{T x_{n}, T x^{*}} \leq m\left(T x_{n}, T x^{*}\right) \rightarrow 0$, so by (2) $T x_{n} \rightarrow T x^{*}$.

Equation (10) implies that $m\left(x_{n}, T x_{n}\right) \rightarrow 0$. Since $m_{x_{n}, T x_{n}} \rightarrow 0$, by Lemma 2.2, we get $m\left(x^{*}, T x^{*}\right)=m_{x^{*}, T x^{*}}$.

On the other hand, by Lemma 2.2 and

$$
T x_{n-1}=x_{n} \rightarrow x^{*} \quad \text { and also } \quad x_{n+1}=T x_{n} \rightarrow T x^{*}
$$

we have

$$
\begin{aligned}
0 & =\lim _{n \rightarrow \infty}\left(m\left(x_{n}, T x_{n}\right)-m_{x_{n}, T x_{n}}\right) \\
& =\lim _{n \rightarrow \infty}\left(m\left(x_{n}, x_{n-1}\right)-m_{x_{n}, T x_{n}}\right) \\
& =m\left(x^{*}, x^{*}\right)-m_{x^{*}, T x^{*}} \\
& =m\left(T x^{*}, T x^{*}\right)-m_{x^{*}, T x^{*}}
\end{aligned}
$$

thus $m\left(x^{*}, x^{*}\right)=m_{x^{*}, T x^{*}}=m\left(T x^{*}, T x^{*}\right)$ and since

$$
m\left(x^{*}, T x^{*}\right)=m_{x^{*}, T x^{*}}=m\left(T x^{*}, T x^{*}\right)=m\left(x^{*}, x^{*}\right)
$$

now by $(\mathrm{m} 1) x^{*}=T x^{*}$. Uniqueness by the contraction (8) is clear. 
Put

$$
C(x, y)=m(x, y)+\frac{(1+m(x, T x)) m(y, T y)}{1+m(x, y)}+\frac{m(x, T x) m(y, T y)}{m(x, y)} .
$$

Theorem 3.2 Let $(X, m)$ be a complete $M$-metric space and let $T$ be a continuous mapping from $X$ into itself satisfying the following condition:

$$
\forall \varepsilon>0 \exists \delta>0 \forall x, y \in X \varepsilon \leq k C(x, y)<\varepsilon+\delta \Rightarrow m(T x, T y)<\varepsilon,
$$

for some $0<k<\frac{1}{3}$. Then $T$ has a unique fixed point $u \in X$. Moreover, for all $x \in X$, the sequence $\left\{T_{n}(x)\right\}$ converges to $u$.

Proof We first observe that (14) trivially implies that $T$ is a strict contraction, i.e.,

$$
x \neq y \Rightarrow m(T x, T y)<k C(x, y) .
$$

Let $x_{0} \in X$ and $x_{n}:=T x_{n-1}$ so we have

$$
\begin{aligned}
C\left(x_{n-1}, x_{n}\right) & =m\left(x_{n-1}, x_{n}\right)+\frac{\left(1+m\left(x_{n-1}, x_{n}\right)\right) m\left(x_{n}, x_{n+1}\right)}{1+m\left(x_{n-1}, x_{n}\right)}+\frac{m\left(x_{n-1}, x_{n}\right) m\left(x_{n}, x_{n+1}\right)}{m\left(x_{n-1}, x_{n}\right)} \\
& \leq k\left(m\left(x_{n-1}, x_{n}\right)+2 m\left(x_{n}, x_{n+1}\right)\right) \\
m\left(x_{n}, x_{n+1}\right) & =m\left(T x_{n-1}, T x_{n}\right) \\
& \leq k C\left(x_{n-1}, x_{n}\right) \\
& \leq k\left(m\left(x_{n-1}, x_{n}\right)+2 m\left(x_{n}, x_{n+1}\right)\right),
\end{aligned}
$$

therefore

$$
m\left(x_{n}, x_{n+1}\right) \leq r m\left(x_{n-1}, x_{n}\right)
$$

where $r=\frac{k}{1-2 k}<1$. Now by Lemma 2.5, $\left\{x_{n}\right\}$ is a Cauchy sequence, and by completeness of $X, T x_{n-1}=x_{n} \rightarrow x^{*}$ in $m$ for some $x^{*} \in X$. Since $T$ is a continuous mapping, so $x_{n}=$ $T x_{n-1} \rightarrow T x^{*}$, in $m$ now by Lemma 2.4 we find

$$
\begin{aligned}
& m\left(x^{*}, T x^{*}\right)=m_{x^{*}, T x^{*}}, \\
& 0=\lim _{n \rightarrow \infty}\left(m\left(x_{n}, T x_{n}\right)-m_{x_{n}, T x_{n}}\right)=m\left(x^{*}, x^{*}\right)-m_{x^{*}, T x^{*}}=m\left(T x^{*}, T x^{*}\right)-m_{x^{*}, T x^{*}},
\end{aligned}
$$

by Lemma 2.2 and

$$
m\left(x^{*}, T x^{*}\right)=m_{x^{*}, T x^{*}}=m\left(T x^{*}, T x^{*}\right)=m\left(x^{*}, x^{*}\right) .
$$

So $x^{*}=T x^{*}$. Uniqueness by the contraction (15) is clear.

Corollary 3.1 (Gupta and Saxena [8]) Let $(X, d)$ be a complete metric space and $T$ be a continuous mapping from $X$ into itself. Assume that $T$ satisfies

$$
\forall x, y \in X x \neq y d(T x, T y) \leq k C(x, y)
$$


where $k \in\left(0, \frac{1}{3}\right)$ is a constant. Then $T$ has a unique fixed point $u \in X$. Moreover, for all $x \in X$, the sequence $\left\{T_{n}(x)\right\}$ converges to $u$.

\section{Applications}

In this section, after an idea of Samet et al. [9], we shall state an integral version of the Gupta-Saxena result.

Theorem 4.1 Let $(X, m)$ be an M-metric space and let $T$ be a self-mapping defined on $X$. Assume that there exists a function $\varphi:[0, \infty) \rightarrow[0, \infty)$ satisfying the following:

(1) $\varphi(0)=0$ and $t>0 \Rightarrow \varphi(t)>0$;

(2) $\varphi$ is nondecreasing and right continuous;

(3) for every $\varepsilon>0$, there exists $\delta>0$ such that

$$
\varepsilon \leq \varphi(k C(x, y))<\varepsilon+\delta \Rightarrow \varphi(m(T x, T y))<\varepsilon,
$$

for some $0<k<\frac{1}{3}$ and for all $x, y \in X$ with $x \neq y$.

Then (14) is satisfied.

Proof Fix $\varepsilon>0$, so $\varphi(\varepsilon)>0$. Hence by (17) there exists $\delta_{1}>0$ such that

$$
\forall x, y \in X x \neq y \varphi(\varepsilon) \leq \varphi(k C(x, y))<\varphi(\varepsilon)+\delta_{1} \Rightarrow \varphi(m(T x, T y))<\varphi(\varepsilon) .
$$

According to the right continuity of $\varphi$

$$
\exists \delta>0 \varphi\left(\varepsilon+\delta_{1}\right)<\varphi(\varepsilon)+\delta .
$$

Now for $x, y \in X$ with $x \neq y$ and fixed

$$
\varepsilon \leq k C(x, y)<\varepsilon+\delta
$$

since $\varphi$ is a nondecreasing mapping, we have

$$
\varphi(\varepsilon) \leq \varphi(k C(x, y))<\varphi\left(\varepsilon+\delta_{1}\right)<\varphi(\varepsilon)+\delta .
$$

So we get

$$
\varphi(m(T x, T y))<\varphi(\varepsilon),
$$

which implies that $m(T x, T y)<\varepsilon$.

Corollary 4.1 Let $(X, m)$ be an M-metric space and let $T$ be a self-mapping defined on $X$. Let $h:[0, \infty) \rightarrow[0, \infty)$ be a locally integrable function such that

(1) $t>0 \Rightarrow \int_{0}^{t} h(s) d s>0$;

(2) for every $\varepsilon>0$, there exists $\delta>0$ such that

$$
\frac{1}{k} \varepsilon \leq \int_{0}^{C(x, y)} h(s) d s<\frac{1}{k} \varepsilon+\delta \Rightarrow \int_{0}^{\frac{1}{k} m(T x, T y)} h(s) d s<\frac{1}{k} \varepsilon,
$$

for some $0<k<\frac{1}{3}$ and for all $x, y \in X$ with $x \neq y$.

Then (14) is satisfied. 


\section{Competing interests}

The author declares to have no competing interests.

\section{Acknowledgements}

This research was supported by the Zanjan Branch, Islamic Azad University, Zanjan, Iran. The author would like to acknowledge this support. The author expresses deep gratitude to the referee for his/her valuable comments and suggestions.

Received: 7 July 2015 Accepted: 5 November 2015 Published online: 17 November 2015

\section{References}

1. Aydi, E, Karapınar, E, Vetro, C: On Ekeland's variational principle in partial metric spaces. Appl. Math. Inf. Sci. 9(1), 257-262 (2015)

2. Borwein, JM, Zhu, QJ: Techniques of Variational Analysis. Springer, New York (2005)

3. Matthews, SG: Partial metric topology. Ann. N.Y. Acad. Sci. 728, 183-197 (1994)

4. Haghi, RH, Rezapour, S, Shahzad, N: Be careful on partial metric fixed point results. Topol. Appl. 160(3), $450-454$ (2013)

5. Asadi, A, Karapınar, E, Salimi, P: New extension of $p$-metric spaces with some fixed point results on $M$-metric spaces. J. Inequal. Appl. 2014, 18 (2014)

6. Shatanawi, W, Postolache, M: Coincidence and fixed point results for generalized weak contractions in the sense of Berinde on partial metric spaces. Fixed Point Theory Appl. 2013, 54 (2013)

7. Meir, A, Keeler, E: A theorem on contraction mappings. J. Math. Anal. Appl. 28(1-3), 326-329 (1969)

8. Gupta, AN, Saxena, A: A unique fixed point theorem in metric spaces. Math. Stud. 52, 156-158 (1984)

9. Samet, B, Vetro, C, Yazidi, H: A fixed point theorem for a Meir-Keeler type contraction through rational expression. J. Nonlinear Sci. Appl. 6, 162-169 (2013)

\section{Submit your manuscript to a SpringerOpen ${ }^{\circ}$ journal and benefit from:}

- Convenient online submission

- Rigorous peer review

- Immediate publication on acceptance

- Open access: articles freely available online

- High visibility within the field

- Retaining the copyright to your article 